\title{
Preface: special issue to commemorate the "International Symposium on Photocatalysis" at Fuzhou University
}

\author{
Xianzhi $\mathbf{F u}^{1}$
}

Received: 25 March 2017/Accepted: 16 April 2017/Published online: 7 August 2017

(C) Springer Science+Business Media B.V. 2017

The International Symposium on Photocatalysis was held at Fuzhou University, China, on November 7th, 2015 with the participation of many famous invited speakers from Asia. As chair of this international symposium and president of Fuzhou University, I am honored to write the preface for this special issue of the international journal, Research on Chemical Intermediates (Springer) to commemorate this symposium. This meeting was organized to celebrate the designation in 2014 of the Research Institute of Photocatalysis of Fuzhou University as a "State Key Laboratory of Photocatalysis on Energy and Environment", the only State Key Laboratory devoted to photocatalysis in China.

Xianzhi Fu

xzfu@fzu.edu.cn

1 Research Institute of Photocatalysis, Fuzhou University, Academician of Chinese Academy of Engineering and Scholar of Photocatalysis, Fuzhou, China 


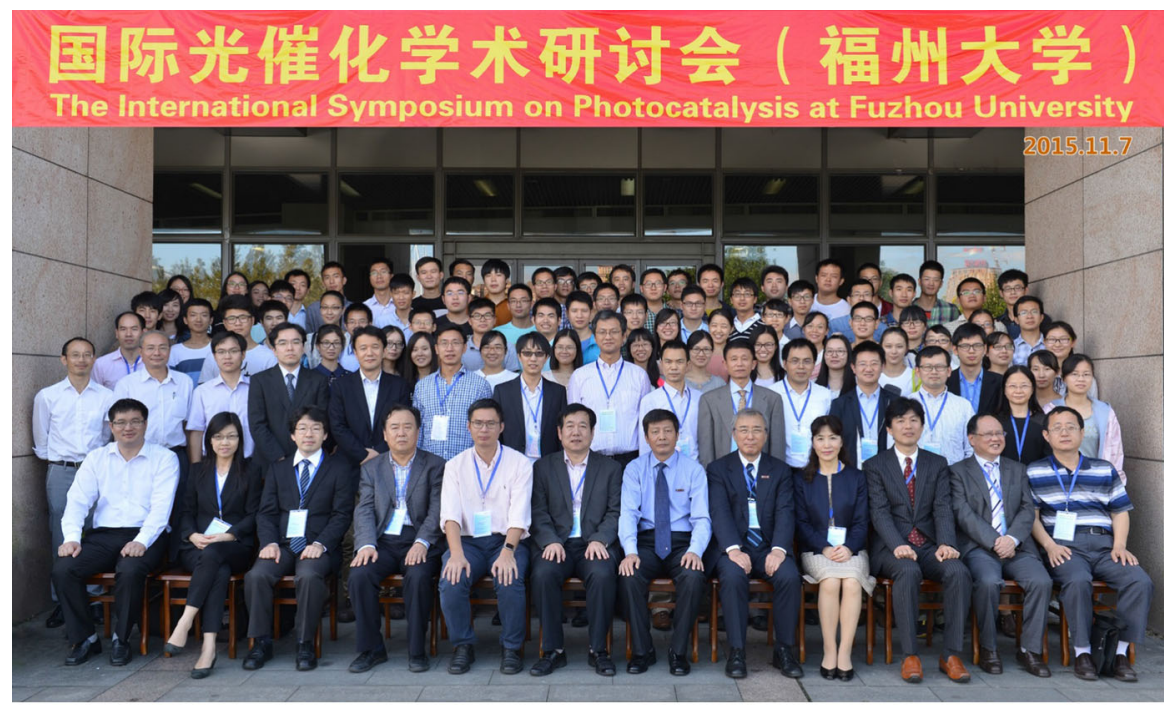

Group photo (photo 1)

A brief historical overview of the Research Institute of Photocatalysis, which was established in 1997 to promote innovative research on environmental issues and clean energy, is shown in Fig. 1. As director of the institute, I have worked with our research teams, which in the beginning consisted of five faculties, to develop and expand research on photocatalytic water-splitting, environmental remediation, photocatalytic organic transformations, and their application in various important areas. We have also been awarded funding for many projects by national and provincial governments including 973 projects supported by the Chinese National Programs for Science and Technology Development, the Ministry of Science and Technology, and the Key Project of the National Natural Science Foundation of China in cooperation with industry and the military. To date, we have published over 500 papers and been issued over 60 patents. I am filled with deep emotion when recalling the nostalgic early days when my good friend Prof. Jincai Zhao, who is an academician of the Chinese Academy of Science and a scholar in photocatalysis, and I worked for several days to complete the 1st key project on photocatalysis of the National Natural Science Foundation of China.

In order to raise international awareness of our institute as one of the largest devoted to photocatalysis in the world and where various innovative and advanced research is being carried out, we have organized this International Symposium on Photocatalysis. This special issue commemorating the symposium should be of great interest to the scientific community since famous scholars and researchers in 


\section{Overview of the Research Institute of Photocatalysis Fuzhou University}

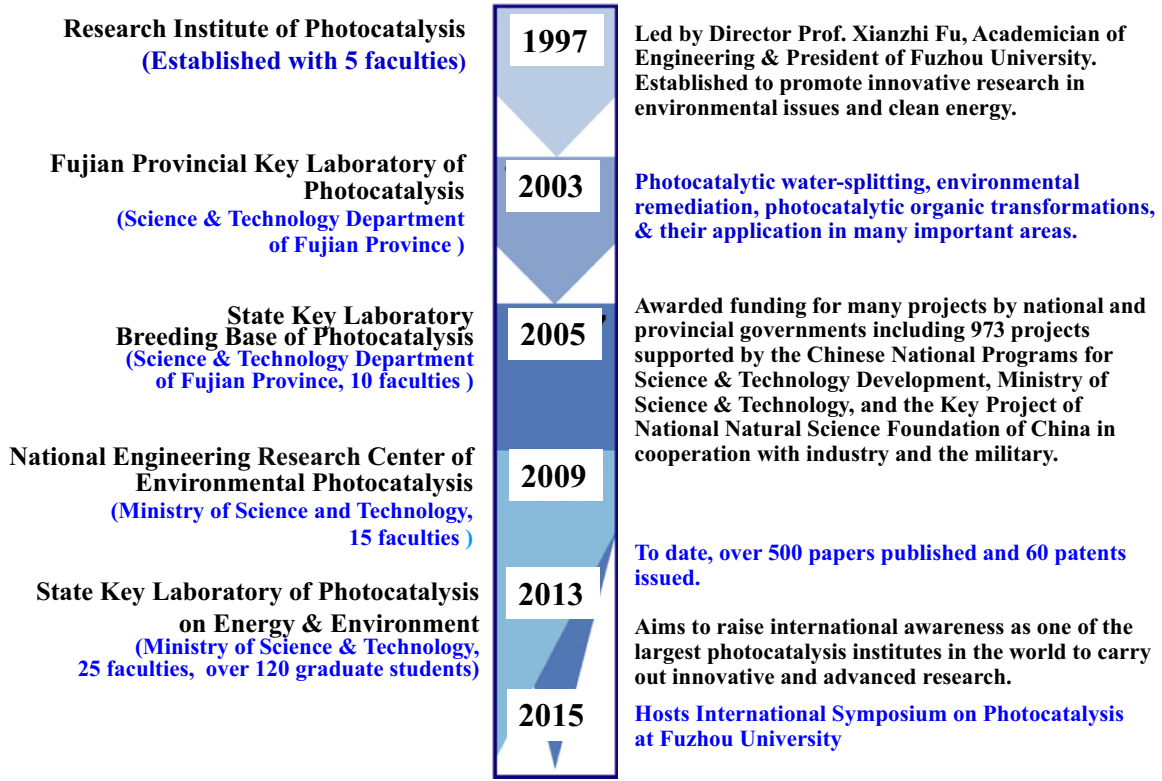

Fig. 1 Brief historical overview of the research institute of photocatalysis, Fuzhou University

photocatalysis from Asia, Europe, and the USA have been invited to contribute. Thus, it includes many interesting original manuscripts and review papers covering such recent topics as electron and proton transfer on semiconductor interfaces, the degradation and remediation of environmental pollution, the production of clean energy, water splitting to produce hydrogen, photofunctional porous materials including metal-organic frameworks (MOF), and other such topical and important themes in photocatalysis.

Finally, I would like to express my sincere appreciation to the guest editors of this special issue, Profs. Xinchen Wang and Zhaohui Li of the State Key Laboratory of Photocatalysis on Energy and Environment of Fuzhou University (China), Prof. Masaya Matsuoka of Osaka Prefecture University (Japan), and Dr. Jinhua Ye of the National Institute for Materials Science (Tsukuba, Japan). Sincere thanks are also extended to Prof. Masakazu Anpo, Editor-in-Chief of Res. Chem. Intermed. and to Springer Publishers for realizing this special issue. Most of all, the great success of the symposium was due to the many participants, and I am deeply grateful for their strong support. 


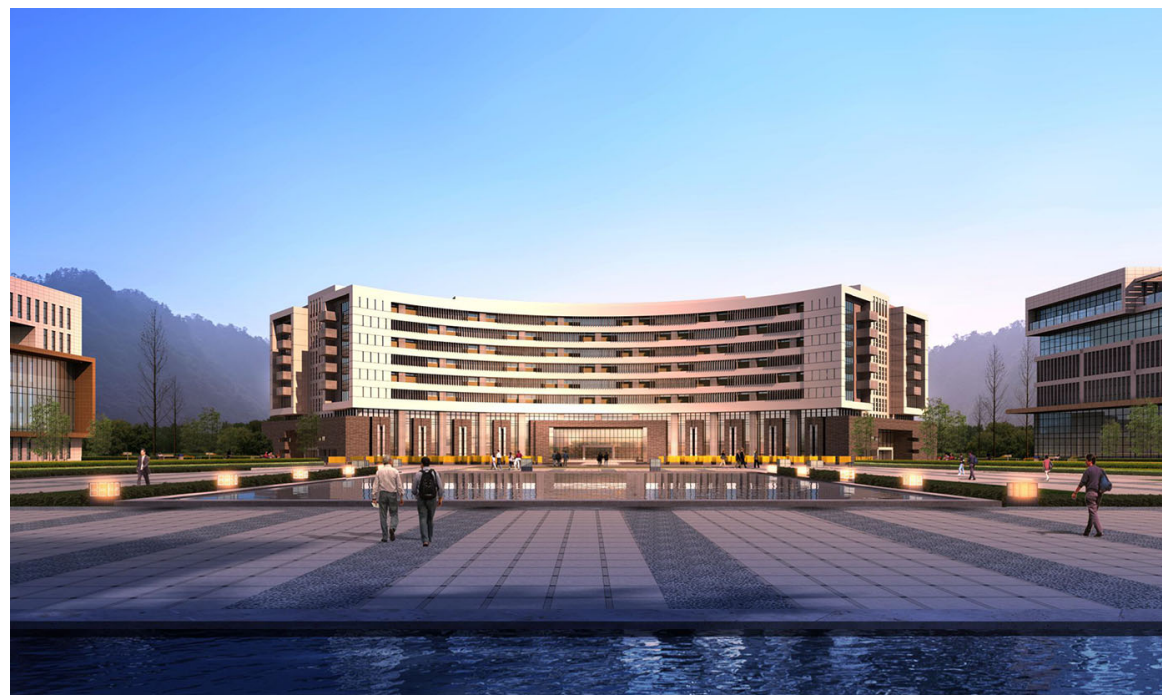

Building of Research Institute of Photocatalysis which occupies 3rd and 4th floors.

We hope this special issue will be of interest and instruction to students and researchers in photocatalysis, as well as inspire the reader community to visit Fuzhou University and the Research Institute of Photocatalysis. We look forward to welcoming you to discuss our common interests and establish future collaborations.

Thank you very much 